\title{
First evidence of hard scattering processes in single tagged $\gamma \gamma$ collisions
}

DELPHI Collaboration

\begin{abstract}
For the first time, multihadronic production from single tagged $\gamma \gamma$ collisions has been studied, where one of the scattered leptons was tagged at very low virtual photon absolute mass squared $\left.\left(<Q^{2}\right\rangle=0.06\left(\mathrm{GeV} / c^{2}\right)^{2}\right)$. Data collected during 1991 and 1992 in the DELPHI experiment at LEP are shown to agree well with predictions which included the non-perturbative vector meson dominance model in which the interacting photons are assumed to have converted into a vector meson $(\rho, \omega$ or $\phi)$, a quark-parton model which describes direct photon interactions and a QCD-based model which considers the photon to have quark and gluon structure functions. Five different parametrizations of these structure functions were used and the predictions compared with the data. This study confirms recent results from no-tag experiments in requiring a QCD-based component to successfully describe the data, indicating that the photon has a significant partonic content.
\end{abstract}


P.Abreu ${ }^{20}$, W.Adam ${ }^{7}$, T.Adye ${ }^{37}$, E.Agasi ${ }^{30}$, I.Ajinenko ${ }^{42}$, R.Aleksan ${ }^{39}$, G.D.Alekseev ${ }^{14}$, P.P.Allport ${ }^{21}$, S.Almehed $^{23}$, F.M.L.Almeida ${ }^{47}$, S.J.Alvsvaag ${ }^{4}$, U.Amaldi ${ }^{7}$, A.Andreazza ${ }^{27}$, M.L.Andrieux ${ }^{12}$, P.Antilogus ${ }^{24}$, W-D.Apel ${ }^{15}$, Y.Arnoud ${ }^{39}$, B.Asman ${ }^{44}$, J-E.Augustin ${ }^{18}$, A.Augustinus ${ }^{30}$, P.Baillon ${ }^{7}$, P.Bambade $^{18}$, F.Barao $^{20}$, R.Barate $^{12}$, G.Barbiellini ${ }^{46}$, D.Y.Bardin ${ }^{14}$, G.J.Barker ${ }^{34}$, A.Baroncelli ${ }^{40}$, O.Barring ${ }^{7}$, J.A.Barrio ${ }^{25}$, W.Bartl ${ }^{50}$, M.J.Bates ${ }^{37}$, M.Battaglia ${ }^{13}$, M.Baubillier ${ }^{22}$, J.Baudot ${ }^{39}$, K-H.Becks ${ }^{52}$, M.Begalli ${ }^{36}$, P.Beilliere ${ }^{6}$, P.Beltran ${ }^{9}$, A.C.Benvenuti $^{5}$, M.Berggren ${ }^{41}$, D.Bertrand ${ }^{2}$, F.Bianchi ${ }^{45}$, M.Bigi ${ }^{45}$, M.S.Bilenky ${ }^{14}$, P.Billoir ${ }^{22}$, J.Bjarne ${ }^{23}$, D.Bloch $^{8}$, S.Blyth ${ }^{34}$, V.Bocci ${ }^{38}$, P.N.Bogolubov ${ }^{14}$, T.Bolognese $^{39}$, M.Bonesini ${ }^{27}$, W.Bonivento ${ }^{27}$, P.S.L.Booth ${ }^{21}$, G.Borisov $^{42}$, C.Bosio ${ }^{40}$, B.Bostjancic ${ }^{43}, \quad$ S.Bosworth $^{34}$, O.Botner ${ }^{48}$, B.Bouquet ${ }^{18}$, C.Bourdarios $^{18}$, T.J.V.Bowcock ${ }^{21}$, M.Bozzo $^{11}$, S.Braibant ${ }^{2}$, P.Branchini ${ }^{40}$, K.D.Brand ${ }^{35}$, R.A.Brenner ${ }^{13}$, H.Briand ${ }^{22}$, C.Bricman ${ }^{2}$, L.Brillault ${ }^{22}$, R.C.A.Brown ${ }^{7}$, J-M.Brunet ${ }^{6}$, L.Bugge ${ }^{32}$, T.Buran ${ }^{32}$, A.Buys $^{7}$, M.Caccia $^{27}$, M.Calvi ${ }^{27}$, A.J.Camacho Rozas ${ }^{41}$, T.Camporesi ${ }^{7}$, V.Canale ${ }^{38}$, M.Canepa $^{11}$, K.Cankocak ${ }^{44}$, F.Cao $^{2}$, F.Carena $^{7}$, P.Carrilho $^{47}$, L.Carroll ${ }^{21}$, C.Caso ${ }^{11}$, V.Cassio ${ }^{45}$, M.V.Castillo Gimenez ${ }^{49}$, A.Cattai ${ }^{7}$, F.R.Cavallo ${ }^{5}$, L.Cerrito $^{38}{ }^{3}$, V.Chabaud ${ }^{7}$, A.Chan ${ }^{1}$, Ph.Charpentier ${ }^{7}$, L.Chaussard ${ }^{24}$, J.Chauveau ${ }^{22}$, P.Checchia ${ }^{35}$, G.A.Chelkov ${ }^{14}$, P.Chliapnikov $^{42}$, V.Chorowicz ${ }^{7}$, J.T.M.Chrin ${ }^{49}$, V.Cindro ${ }^{43}$, P.Collins ${ }^{34}$, J.L.Contreras ${ }^{18}$, R.Contri ${ }^{11}$, E.Cortina $^{49}$, G.Cosme ${ }^{18}$, F.Couchot ${ }^{18}$, H.B.Crawley ${ }^{1}$, D.Crennell ${ }^{37}$, G.Crosetti ${ }^{11}$, J.Cuevas Maestro ${ }^{33}$, S.Czellar ${ }^{13}$, E.Dahl-Jensen ${ }^{28}$, J.Dahm ${ }^{52}$, B.Dalmagne ${ }^{18}$, M.Dam ${ }^{32}$, G.Damgaard ${ }^{28}$, E.Daubie ${ }^{2}$, A.Daum ${ }^{15}$, P.D.Dauncey ${ }^{37}$, M.Davenport ${ }^{7}$, W.Da Silva ${ }^{22}$, C.Defoix ${ }^{6}$, P.Delpierre ${ }^{26}$, N.Demaria ${ }^{34}$, A.De Angelis ${ }^{7}$, H.De Boeck ${ }^{2}$, W.De Boer ${ }^{15}$, S.De Brabandere ${ }^{2}$, C.De Clercq ${ }^{2}$, M.D.M.De Fez Laso ${ }^{49}$, C.De La Vaissiere ${ }^{22}$, B.De Lotto ${ }^{46}$, A.De Min ${ }^{27}$, L.De Paula ${ }^{47}$, C.De Saint-Jean ${ }^{39}$, H.Dijkstra ${ }^{7}$, L.Di Ciaccio ${ }^{38}$, F.Djama ${ }^{8}$, J.Dolbeau $^{6}$, M.Donszelmann ${ }^{7}$, K.Doroba ${ }^{51}$, M.Dracos $^{8}$, J.Drees ${ }^{52}$, M.Dris ${ }^{31}$, Y.Dufour ${ }^{6}$, F.Dupont ${ }^{12}$, D.Edsall ${ }^{1}$, R.Ehret $^{15}$, T.Ekelof ${ }^{48}$, G.Ekspong ${ }^{44}$, M.Elsing ${ }^{52}$, J-P.Engel ${ }^{8}$, N.Ershaidat ${ }^{22}$, M.Espirito Santo ${ }^{20}$, D.Fassouliotis ${ }^{31}$, M.Feindt ${ }^{7}$, A.Ferrer ${ }^{49}$, T.A.Filippas ${ }^{31}$, A.Firestone ${ }^{1}$, H.Foeth ${ }^{7}$, E.Fokitis ${ }^{31}$, F.Fontanelli ${ }^{11}$, F.Formenti $^{7}$, J-L.Fousset ${ }^{26}$, B.Franek ${ }^{37}$, P.Frenkiel ${ }^{6}$, D.C.Fries ${ }^{15}$, A.G.Frodesen ${ }^{4}$, R.Fruhwirth ${ }^{50}$, F.Fulda-Quenzer ${ }^{18}$, H.Furstenau ${ }^{7}$, J.Fuster ${ }^{7}$, D.Gamba ${ }^{45}$, M.Gandelman ${ }^{17}$, C.Garcia ${ }^{49}$, J.Garcia ${ }^{41}$, C.Gaspar $^{7}$, U.Gasparini $^{35}$, Ph.Gavillet ${ }^{7}$, E.N.Gazis ${ }^{31}$, D.Gele ${ }^{8}$, J-P.Gerber ${ }^{8}$, D.Gillespie ${ }^{7}$, R.Gokieli ${ }^{51}$, B.Golob ${ }^{43}$, V.M.Golovatyuk ${ }^{14}$, J.J.Gomez Y Cadenas ${ }^{7}$, G.Gopal ${ }^{37}$, L.Gorn ${ }^{1}$, M.Gorski ${ }^{51}$, V.Gracco ${ }^{11}$, F.Grard ${ }^{2}$, E.Graziani ${ }^{40}$, G.Grosdidier ${ }^{18}$, P.Gunnarsson ${ }^{44}$, J.Guy ${ }^{37}$, U.Haedinger ${ }^{15}$, F.Hahn ${ }^{52}$, M.Hahn ${ }^{15}$, S.Hahn ${ }^{52}$, S.Haider $^{30}$, Z.Hajduk ${ }^{16}$, A.Hakansson ${ }^{23}$, A.Hallgren ${ }^{48}$, K.Hamacher ${ }^{52}$, W.Hao ${ }^{30}$, F.J.Harris ${ }^{34}$, V.Hedberg ${ }^{23}$, R.Henriques $^{20}$, J.J.Hernandez ${ }^{49}$, J.A.Hernando ${ }^{49}$, P.Herquet ${ }^{2}$, H.Herr ${ }^{7}$, T.L.Hessing ${ }^{7}$, E.Higon ${ }^{49}$, H.J.Hilke ${ }^{7}$, T.S.Hill $^{1}$, S-O.Holmgren ${ }^{4}$, P.J.Holt ${ }^{34}$, D.Holthuizen ${ }^{30}$, P.F.Honore ${ }^{6}$, M.Houlden ${ }^{21}$, J.Hrubec ${ }^{50}$, K.Huet ${ }^{2}$, K.Hultqvist $^{44}$, P.Ioannou ${ }^{3}$, P-S.Iversen ${ }^{4}$, J.N.Jackson ${ }^{21}$, R.Jacobsson ${ }^{44}$, P.Jalocha ${ }^{16}$, G.Jarlskog $^{23}$, P.Jarry ${ }^{39}$, B.Jean-Marie ${ }^{18}$, E.K.Johansson ${ }^{44}$, L.Jonsson ${ }^{23}$, P.Juillot ${ }^{8}$, M.Kaiser ${ }^{15}$, G.Kalmus ${ }^{37}$, F.Kapusta ${ }^{22}$, M.Karlsson $^{44}$, E.Karvelas ${ }^{9}$, A.Katargin ${ }^{42}$, S.Katsanevas ${ }^{3}$, E.C.Katsoufis ${ }^{31}$, R.Keranen ${ }^{13}$, B.A.Khomenko ${ }^{14}$, N.N.Khovanski ${ }^{14}$, B.King ${ }^{21}$, N.J.Kjaer ${ }^{28}$, H.Klein ${ }^{7}$, A.Klovning ${ }^{4}$, P.Kluit ${ }^{30}$, A.Koch-Mehrin ${ }^{52}$, J.H.Koehne ${ }^{15}$, B.Koene $^{30}$, P.Kokkinias ${ }^{9}$, M.Koratzinos ${ }^{7}$, K.Korcyl ${ }^{16}$, A.V.Korytov ${ }^{14}$, V.Kostioukhine ${ }^{42}$, C.Kourkoumelis ${ }^{3}$, O.Kouznetsov $^{11}$, P.-H.Kramer ${ }^{52}$, C.Kreuter ${ }^{15}$, J.Krolikowski ${ }^{51}$, I.Kronkvist ${ }^{23}$, W.Krupinski ${ }^{16}$, K.Kulka ${ }^{48}$, K.Kurvinen $^{13}$, C.Lacasta ${ }^{49}$, I.Laktineh ${ }^{24}$, C.Lambropoulos ${ }^{9}$, J.W.Lamsa ${ }^{1}$, L.Lanceri ${ }^{46}$, P.Langefeld $^{52}$, V.Lapin ${ }^{42}$, I.Last ${ }^{21}$, J-P.Laugier ${ }^{39}$, R.Lauhakangas ${ }^{13}$, G.Leder ${ }^{50}$, F.Ledroit ${ }^{12}$, R.Leitner ${ }^{29}$, Y.Lemoigne ${ }^{39}$, J.Lemonne $^{2}$, G.Lenzen ${ }^{52}$, V.Lepeltier ${ }^{18}$, T.Lesiak ${ }^{35}$, J.M.Levy ${ }^{8}$, E.Lieb ${ }^{52}$, D.Liko ${ }^{50}$, R.Lindner ${ }^{52}$, A.Lipniacka $^{18}$, I.Lippi $^{35}$, B.Loerstad ${ }^{23}$, M.Lokajicek ${ }^{10}$, J.G.Loken ${ }^{34}$, A.Lopez-Fernandez ${ }^{7}$, M.A.Lopez Aguera ${ }^{41}$, M.Los $^{30}$, D.Loukas ${ }^{9}$, J.J.Lozano ${ }^{49}$, P.Lutz ${ }^{39}$, L.Lyons ${ }^{34}$, G.Maehlum ${ }^{15}$, J.Maillard ${ }^{6}$, A.Maio ${ }^{20}$, A.Maltezos ${ }^{9}$, F.Mandl ${ }^{50}$, J.Marco ${ }^{41}$, B.Marechal ${ }^{47}$, M.Margoni ${ }^{35}$, J-C.Marin ${ }^{7}$, C.Mariotti ${ }^{40}$, A.Markou ${ }^{9}$, T.Maron $^{52}$, S.Marti $^{49}$, C.Martinez-Rivero ${ }^{41}$, F.Martinez-Vidal ${ }^{49}$, F.Matorras ${ }^{41}$, C.Matteuzzi ${ }^{27}$, G.Matthiae ${ }^{38}$, M.Mazzucato $^{35}$, M.Mc Cubbin ${ }^{7}$, R.Mc Kay ${ }^{1}$, R.Mc Nulty ${ }^{21}$, J.Medbo ${ }^{48}$, C.Meroni ${ }^{27}$, W.T.Meyer ${ }^{1}$, A.Miagkov $^{42}$, M.Michelotto ${ }^{35}$, E.Migliore ${ }^{45}$, I.Mikulec ${ }^{50}$, L.Mirabito ${ }^{24}$, W.A.Mitaroff ${ }^{50}$, G.V.Mitselmakher ${ }^{14}$, U.Mjoernmark $^{23}$, T.Moa ${ }^{44}$, R.Moeller ${ }^{28}$, K.Moenig ${ }^{7}$, M.R.Monge ${ }^{11}$, P.Morettini ${ }^{11}$, H.Mueller ${ }^{15}$, W.J.Murray ${ }^{37}$, B.Muryn $^{16}$, G.Myatt ${ }^{34}$, F.Naraghi ${ }^{12}$, F.L.Navarria ${ }^{5}$, S.Navas ${ }^{49}$, P.Negri ${ }^{27}$, S.Nemecek ${ }^{10}$, W.Neumann ${ }^{52}$, N.Neumeister $^{50}$, R.Nicolaidou ${ }^{3}$, B.S.Nielsen ${ }^{28}$, V.Nikolaenko ${ }^{24}$, P.Niss ${ }^{44}$, A.Nomerotski ${ }^{35}$, A.Normand ${ }^{34}$, V.Obraztsov ${ }^{42}$, A.G.Olshevski ${ }^{14}$, R.Orava ${ }^{13}$, K.Osterberg ${ }^{13}$, A.Ouraou ${ }^{39}$, P.Paganini ${ }^{18}$, M.Paganoni ${ }^{27}$, R.Pain $^{22}$, H.Palka ${ }^{16}$, Th.D.Papadopoulou ${ }^{31}$, L.Pape ${ }^{7}$, F.Parodi ${ }^{11}$, A.Passeri ${ }^{40}$, M.Pegoraro ${ }^{35}$, J.Pennanen ${ }^{13}$, L.Peralta $^{20}$, H.Pernegger ${ }^{50}$, M.Pernicka ${ }^{50}$, A.Perrotta ${ }^{5}$, C.Petridou ${ }^{46}$, A.Petrolini ${ }^{11}$, H.T.Phillips ${ }^{37}$, G.Piana ${ }^{11}$, F.Pierre $^{39}$, M.Pimenta ${ }^{20}$, S.Plaszczynski ${ }^{18}$, O.Podobrin ${ }^{15}$, M.E.Pol ${ }^{17}$, G.Polok ${ }^{16}$, P.Poropat ${ }^{46}$, V.Pozdniakov $^{14}{ }^{4}$, M.Prest $^{46}$, P.Privitera ${ }^{38}$, A.Pullia ${ }^{27}$, D.Radojicic ${ }^{34}$, S.Ragazzi ${ }^{27}$, H.Rahmani ${ }^{31}$, J.Rames ${ }^{10}$, P.N.Ratoff ${ }^{19}$, A.L.Read ${ }^{32}$, M.Reale ${ }^{52}$, P.Rebecchi ${ }^{18}$, N.G.Redaelli ${ }^{27}$, M.Regler ${ }^{50}$, D.Reid ${ }^{7}$, P.B.Renton ${ }^{34}$, L.K.Resvanis ${ }^{3}$, F.Richard ${ }^{18}$, J.Richardson ${ }^{21}$, J.Ridky ${ }^{10}$, G.Rinaudo ${ }^{45}$, I.Ripp ${ }^{39}$, A.Romero ${ }^{45}$, I.Roncagliolo ${ }^{11}$, P.Ronchese ${ }^{35}$, L.Roos $^{12}$, E.I.Rosenberg ${ }^{1}$, E.Rosso ${ }^{7}$, P.Roudeau ${ }^{18}$, T.Rovelli ${ }^{5}$, W.Ruckstuhl ${ }^{30}$, V.Ruhlmann-Kleider ${ }^{39}$, A.Ruiz ${ }^{41}$, K.Rybicki ${ }^{16}$, H.Saarikko ${ }^{13}$, Y.Sacquin ${ }^{39}$, G.Sajot ${ }^{12}$, J.Salt ${ }^{49}$, J.Sanchez ${ }^{25}$, M.Sannino ${ }^{11}$, H.Schneider ${ }^{15}$, M.A.E.Schyns ${ }^{52}$, G.Sciolla ${ }^{45}$, F.Scuri ${ }^{46}$, A.M.Segar ${ }^{34}$, A.Seitz ${ }^{15}$, R.Sekulin ${ }^{37}$, M.Sessa $^{46}$, R.Seufert ${ }^{15}$, R.C.Shellard ${ }^{36}$, I.Siccama ${ }^{30}$, P.Siegrist ${ }^{39}$, S.Simonetti ${ }^{39}$, F.Simonetto ${ }^{35}$, A.N.Sisakian ${ }^{14}$, T.B.Skaali ${ }^{32}$, G.Smadja ${ }^{24}$, O.Smirnova ${ }^{14}$, G.R.Smith ${ }^{37}$, A.Sokolov ${ }^{42}$, R.Sosnowski ${ }^{51}$, D.Souza-Santos ${ }^{36}$, T.Spassov ${ }^{20}$, E.Spiriti ${ }^{40}$, S.Squarcia ${ }^{11}$, H.Staeck ${ }^{52}$, C.Stanescu ${ }^{40}$, S.Stapnes ${ }^{32}$, I.Stavitski ${ }^{35}$, G.Stavropoulos ${ }^{9}$, K.Stepaniak $^{51}$, F.Stichelbaut ${ }^{7}$, A.Stocchi ${ }^{18}$, J.Strauss ${ }^{50}$, J.Straver ${ }^{7}$, R.Strub ${ }^{8}$, B.Stugu ${ }^{4}$, M.Szczekowski ${ }^{51}$, M.Szeptycka $^{51}$, T.Tabarelli ${ }^{27}$, O.Tchikilev ${ }^{42}$, G.E.Theodosiou ${ }^{9}$, Z.Thome ${ }^{47}$, A.Tilquin ${ }^{26}$, J.Timmermans ${ }^{30}$, 
V.G.Timofeev ${ }^{14}$, L.G.Tkatchev ${ }^{14}$, T.Todorov ${ }^{8}$, D.Z.Toet ${ }^{30}$, A.Tomaradze ${ }^{2}$, B.Tome ${ }^{20}$, E.Torassa ${ }^{45}$, L.Tortora $^{40}$,

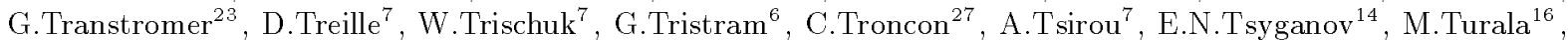
M-L.Turluer $^{39}$, T.Tuuva ${ }^{13}$, I.A.Tyapkin ${ }^{22}$, M.Tyndel ${ }^{37}$, S.Tzamarias ${ }^{21}$, B.Ueberschaer ${ }^{52}$, S.Ueberschaer ${ }^{52}$, O.Ullaland $^{7}$, V.Uvarov ${ }^{42}$, G.Valenti ${ }^{5}$, E.Vallazza ${ }^{7}$, J.A.Valls Ferrer ${ }^{49}$, C.Vander Velde ${ }^{2}$, G.W.Van Apeldoorn ${ }^{30}$, P.Van Dam ${ }^{30}$, W.K.Van Doninck ${ }^{2}$, J.Van Eldik ${ }^{30}$, G.Vegni ${ }^{27}$, L.Ventura ${ }^{35}$, W.Venus ${ }^{37}$, F.Verbeure ${ }^{2}$, M.Verlato $^{35}$, L.S.Vertogradov ${ }^{14}$, D.Vilanova ${ }^{39}$, P.Vincent ${ }^{24}$, L.Vitale ${ }^{46}$, E.Vlasov ${ }^{42}$, A.S.Vodopyanov ${ }^{14}$, M.Vollmer ${ }^{52}$, M.Voutilainen ${ }^{13}$, V.Vrba ${ }^{10}$, H.Wahlen $^{52}$, C.Walck $^{44}$, F.Waldner ${ }^{46}$, A.Wehr ${ }^{52}$, M.Weierstall ${ }^{52}$, P.Weilhammer $^{7}$, A.M.Wetherell ${ }^{7}$, J.H.Wickens ${ }^{2}$, M.Wielers ${ }^{15}$, G.R.Wilkinson ${ }^{34}$, W.S.C.Williams ${ }^{34}$, M.Winter ${ }^{8}$, M.Witek $^{7}$, G.Wormser ${ }^{18}$, K.Woschnagg ${ }^{48}$, K.Yip ${ }^{34}$, O.Yushchenko ${ }^{42}$, F.Zach ${ }^{24}$, A.Zaitsev ${ }^{42}$, A.Zalewska ${ }^{16}$, P.Zalewski $^{51}$, D.Zavrtanik ${ }^{43}$, E.Zevgolatakos ${ }^{9}$, N.I.Zimin ${ }^{14}$, M.Zito ${ }^{39}$, D.Zontar ${ }^{43}$, R.Zuberi ${ }^{34}$, G.C.Zucchelli ${ }^{44}$, G.Zumerle ${ }^{35}$

\footnotetext{
${ }^{1}$ Ames Laboratory and Department of Physics, Iowa State University, Ames IA 50011, USA

${ }^{2}$ Physics Department, Univ. Instelling Antwerpen, Universiteitsplein 1, B-2610 Wilrijk, Belgium and IIHE, ULB-VUB, Pleinlaan 2, B-1050 Brussels, Belgium

and Faculté des Sciences, Univ. de l'Etat Mons, Av. Maistriau 19, B-7000 Mons, Belgium

${ }^{3}$ Physics Laboratory, University of Athens, Solonos Str. 104, GR-10680 Athens, Greece

${ }^{4}$ Department of Physics, University of Bergen, Allégaten 55, N-5007 Bergen, Norway

${ }^{5}$ Dipartimento di Fisica, Università di Bologna and INFN, Via Irnerio 46, I-40126 Bologna, Italy

${ }^{6}$ Collège de France, Lab. de Physique Corpusculaire, IN2P3-CNRS, F-75231 Paris Cedex 05, France

${ }^{7}$ CERN, CH-1211 Geneva 23, Switzerland

${ }^{8}$ Centre de Recherche Nucléaire, IN2P3 - CNRS/ULP - BP20, F-67037 Strasbourg Cedex, France

${ }^{9}$ Institute of Nuclear Physics, N.C.S.R. Demokritos, P.O. Box 60228, GR-15310 Athens, Greece

${ }^{10}$ FZU, Inst. of Physics of the C.A.S. High Energy Physics Division, Na Slovance 2, 180 40, Praha 8, Czech Republic

${ }^{11}$ Dipartimento di Fisica, Università di Genova and INFN, Via Dodecaneso 33, I-16146 Genova, Italy

${ }^{12}$ Institut des Sciences Nucléaires, IN2P3-CNRS, Université de Grenoble 1, F-38026 Grenoble Cedex, France

${ }^{13}$ Research Institute for High Energy Physics, SEFT, P.O. Box 9, FIN-00014 Helsinki, Finland

${ }^{14}$ Joint Institute for Nuclear Research, Dubna, Head Post Office, P.O. Box 79, 101000 Moscow, Russian Federation

${ }^{15}$ Institut für Experimentelle Kernphysik, Universität Karlsruhe, Postfach 6980, D-76128 Karlsruhe, Germany

${ }^{16}$ High Energy Physics Laboratory, Institute of Nuclear Physics, Ul. Kawiory 26a, PL-30055 Krakow 30, Poland

${ }^{17}$ Centro Brasileiro de Pesquisas Físicas, rua Xavier Sigaud 150, BR-22290 Rio de Janeiro, Brazil

${ }^{18}$ Université de Paris-Sud, Lab. de l'Accélérateur Linéaire, IN2P3-CNRS, Bat 200, F-91405 Orsay Cedex, France

${ }^{19}$ School of Physics and Materials, University of Lancaster, Lancaster LA1 4YB, UK

${ }^{20}$ LIP, IST, FCUL - Av. Elias Garcia, 14-1 ${ }^{\circ}$, P-1000 Lisboa Codex, Portugal

${ }^{21}$ Department of Physics, University of Liverpool, P.O. Box 147, Liverpool L69 3BX, UK

${ }^{22}$ LPNHE, IN2P3-CNRS, Universités Paris VI et VII, Tour 33 (RdC), 4 place Jussieu, F-75252 Paris Cedex 05, France

${ }^{23}$ Department of Physics, University of Lund, Sölvegatan 14, S-22363 Lund, Sweden

${ }^{24}$ Université Claude Bernard de Lyon, IPNL, IN2P3-CNRS, F-69622 Villeurbanne Cedex, France

${ }^{25}$ Universidad Complutense, Avda. Complutense s/n, E-28040 Madrid, Spain

${ }^{26}$ Univ. d'Aix - Marseille II - CPP, IN2P3-CNRS, F-13288 Marseille Cedex 09, France

${ }^{27}$ Dipartimento di Fisica, Università di Milano and INFN, Via Celoria 16, I-20133 Milan, Italy

${ }^{28}$ Niels Bohr Institute, Blegdamsvej 17, DK-2100 Copenhagen 0, Denmark

${ }^{29} \mathrm{NC}$, Nuclear Centre of MFF, Charles University, Areal MFF, V Holesovickach 2, 180 00, Praha 8, Czech Republic

${ }^{30}$ NIKHEF-H, Postbus 41882, NL-1009 DB Amsterdam, The Netherlands

${ }^{31}$ National Technical University, Physics Department, Zografou Campus, GR-15773 Athens, Greece

${ }^{32}$ Physics Department, University of Oslo, Blindern, N-1000 Oslo 3, Norway

${ }^{33}$ Dpto. Fisica, Univ. Oviedo, C/P. Pérez Casas, S/N-33006 Oviedo, Spain

${ }^{34}$ Department of Physics, University of Oxford, Keble Road, Oxford OX1 3RH, UK

${ }^{35}$ Dipartimento di Fisica, Università di Padova and INFN, Via Marzolo 8, I-35131 Padua, Italy

${ }^{36}$ Depto. de Fisica, Pontificia Univ. Católica, C.P. 38071 RJ-22453 Rio de Janeiro, Brazil

${ }^{37}$ Rutherford Appleton Laboratory, Chilton, Didcot OX11 OQX, UK

${ }^{38}$ Dipartimento di Fisica, Università di Roma II and INFN, Tor Vergata, I-00173 Rome, Italy

${ }^{39}$ Centre d'Etude de Saclay, DSM/DAPNIA, F-91191 Gif-sur-Yvette Cedex, France

${ }^{40}$ Istituto Superiore di Sanità, Ist. Naz. di Fisica Nucl. (INFN), Viale Regina Elena 299, I-00161 Rome, Italy

${ }^{41}$ C.E.A.F.M., C.S.I.C. - Univ. Cantabria, Avda. los Castros, S/N-39006 Santander, Spain, (CICYT-AEN93-0832)

${ }^{42}$ Inst. for High Energy Physics, Serpukov P.O. Box 35, Protvino, (Moscow Region), Russian Federation

${ }^{43} \mathrm{~J}$. Stefan Institute and Department of Physics, University of Ljubljana, Jamova 39, SI-61000 Ljubljana, Slovenia

${ }^{44}$ Fysikum, Stockholm University, Box 6730, S-113 85 Stockholm, Sweden

${ }^{45}$ Dipartimento di Fisica Sperimentale, Università di Torino and INFN, Via P. Giuria 1, I-10125 Turin, Italy

${ }^{46}$ Dipartimento di Fisica, Università di Trieste and INFN, Via A. Valerio 2, I-34127 Trieste, Italy and Istituto di Fisica, Università di Udine, I-33100 Udine, Italy

${ }^{47}$ Univ. Federal do Rio de Janeiro, C.P. 68528 Cidade Univ., Tlha do Fundão BR-21945-970 Rio de Janeiro, Brazil

${ }^{48}$ Department of Radiation Sciences, University of Uppsala, P.O. Box 535, S-751 21 Uppsala, Sweden

${ }^{49}$ IFIC, Valencia-CSIC, and D.F.A.M.N., U. de Valencia, Avda. Dr. Moliner 50, E-46100 Burjassot (Valencia), Spain

${ }^{50}$ Institut für Hochenergiephysik, Österr. Akad. d. Wissensch., Nikolsdorfergasse 18, A-1050 Vienna, Austria

${ }^{51}$ Inst. Nuclear Studies and University of Warsaw, Ul. Hoza 69, PL-00681 Warsaw, Poland

${ }^{52}$ Fachbereich Physik, University of Wuppertal, Postfach 100 127, D-42097 Wuppertal 1, Germany
} 


\section{Introduction}

Two-photon scattering in $e^{+} e^{-}$storage rings has been studied in many experiments at PEP [1], PETRA [2], and recently at KEK [3] and LEP [4-7]. The production of multihadronic final states, $X$, in the reaction $e^{+} e^{-} \rightarrow e^{+} e^{-} X$ is possible due to collisions of the clouds of virtual photons radiated by high energy electrons and positrons. If both the electron and positron in the final state go down the beam pipe and remain undetected, (the no-tag or untagged mode), only the multihadronic system can be studied. In the single-tagged case one of the outgoing scattered leptons is measured while double-tagged events have both measured. The detected lepton provides additional information about the event kinematics, allowing more detailed studies of such events to be made than is possible with untagged events. Other features of the tagging are useful : the data sample is free from $Z^{0}$ contamination and it becomes possible to study and reject most of the LEP machine background. Unfortunately, as the scattered leptons emerge preferentially along the beam direction, requiring that one or both leptons be scattered at a large enough angle to be detected results in a large suppression of the $\gamma \gamma$ cross-section compared with the untagged case. A review of two-photon physics may be found in ref. [8] and recent reports of work in this field may be found in ref. [9]. Interesting results were reported there by the AMY Collaboration [10] for no-tag experimental conditions giving the first satisfactory qualitative description of data by including QCD-based calculations of the hard scattering of hadronic constituents of the photon.

Here, first evidence of hard scattering subprocesses in single tagged $\gamma \gamma$ events is reported and a comparison is made between the data and five different parton density parametrizations used to describe those subprocesses. Data taken with the DELPHI detector during 1991 and 1992, corresponding to an integrated luminosity of $28.4 \mathrm{pb}^{-1}$, were used to analyze the hadronic final states produced in single tagged $\gamma \gamma$ collisions at a mean value of the tagged-photon absolute squared-mass, $Q^{2}$, around $0.06\left(\mathrm{GeV} / c^{2}\right)^{2}$.

The paper is organized in the following way. Section 2 gives a brief overview of the theory of $\gamma \gamma$ interactions, with emphasis on the three models used for describing the data. Then the implementation of the $\gamma \gamma$ theory in simulation is described. The DELPHI detector components and the methods used for $\gamma \gamma$ event selection are described briefly in Section 3. The detector for luminosity measurement, the Very Small Angle Tagger (VSAT), used here to tag the scattered leptons, is described in more detail. Section 4 deals with the rejection of background to the $\gamma \gamma$ events and the results are discussed in Section 5 .

\section{Theoretical framework and simulation}

The overall kinematics of a single tagged $\gamma \gamma$ reaction is represented in Figure 1a. The four-momentum transfer $Q^{2}=-q_{1}^{2}$ is defined as the absolute value of the squared mass of the virtual photon radiated from the tagged electron or positron. In the high $Q^{2}$ region the er Deep Inelastic Scattering (DIS) formalism applies with a quasi-real photon as the target, coupling to quarks in a point-like fashion or through a vector-meson bound-state (see for example [4]). At moderate $x\left(x=Q^{2} /\left(Q^{2}+W^{2}\right)\right)$ where $W$ is the invariant mass of the produced hadronic system) the data from previous $e^{+} e^{-}$collider experiments are in qualitative agreement with a composite model combining the Vector-meson Dominance Model (VDM) and the Quark-Parton Model (QPM), illustrated in Figures 1b and 1c. The photon structure function is then extracted from the data using unfolding methods [6] . The theoretical description of the low- $x$ region is still not fully satisfactory. An excess of 
events over the incoherent sum of the expectations from the VDM and QPM was observed experimentally [1-3]. It was also observed that a significant fraction of those events did not have the simple two-jet topology expected from the model. QCD-based calculations of non-diffractive hard-scattering of hadronic constituents of the photons have shown that high transverse momentum hadrons could be produced in multi-jet configurations [11,12]. In this low- $x$ region, jets are produced with high transverse momentum $\left(p_{T}\right)$ with respect to the $\gamma \gamma$ axis (which is usually very close to the beam axis for the single tag case) which implies high $\gamma \gamma$ invariant masses $W$.

Going to low $Q^{2}$, jet production with $\left(p_{T} / c\right)^{2}$ greater than $Q^{2}$ is expected to be more frequent. In the Leading Log approximation of QCD, the hard scattering subprocesses shown in Figures $1 \mathrm{~d}$ and $1 \mathrm{e}$ start to grow, even to dominate when going to very low $Q^{2}$. In these subprocesses, called sometimes QCD Resolved Photon Contribution (hereafter denoted QCD-RPC), some partons within the photons can interact with each other. Therefore, a density function formalism is appropriate to express the chance of finding a parton in the photon with given momentum fraction. These QCD-RPC subprocesses offer the possibility of experimentally measuring the quark and gluon densities of the photon [3,4] and hence testing the theoretical models. The hard scattering subprocesses shown in Figures $1 d$ and 1e require high four-momentum transfers (or high $p_{T}^{2}$ ) of partons to probe the structure of one or both photons and to resolve them into their partonic constituents.

Thus the scheme used to describe the $\gamma \gamma$ process can be represented as follows. The fully non-perturbative contribution is described through VDM as the diffractive scattering of vector mesons with the cross-section given by Rosner [13]

$$
\sigma_{\gamma \gamma}\left(W^{2}, Q^{2}, P^{2}\right)=F_{V D M}\left(Q^{2}\right) F_{V D M}\left(P^{2}\right)\left[A+\frac{B}{W}\right]
$$

where $W$ is the invariant mass of the $\gamma \gamma$ system and $Q^{2}$ and $P^{2}$ are the absolute squared masses of the two virtual photons. The values $A=275 \mathrm{nb}$ and $B=300 \mathrm{nb} \cdot \mathrm{GeV} / c^{2}$ were used [14], which are about $10 \%$ larger than those in the standard formula [13]. This type of parametrization has already been used by previous experiments [2,3].

The quantity $F_{V D M}$ is the generalized VDM form factor [15]

$$
F_{V D M}\left(Q^{2}\right)=\sum_{V=\rho, \omega, \phi} r_{V} \frac{1+Q^{2} / 4 m_{V}^{2}}{\left(1+Q^{2} / m_{V}^{2}\right)^{2}}+\frac{0.22}{1+Q^{2} / m_{0}^{2}}
$$

with $m_{0}=1.4 \mathrm{GeV} / c^{2}$ and where $m_{V}$ denotes a vector meson mass and $r_{V}$ is related to the vector meson coupling to the photon. The last term describes the contribution from the radial excitations of vector mesons.

In the simulated events the multihadronic final-state was generated from a $q \bar{q}$ pair, with a limited- $p_{T}$ quark distribution $d \sigma / d p_{T}^{2} \simeq \exp \left(-5 p_{T}^{2}\right)$ in the $\gamma \gamma$ centre-of-mass system using the PLUTO tuned parameter [2].

The other contributions are treated using leading order QCD factorization: a hard scattering subprocess gives the dominant scale $p_{T}^{2}$, taken also as the factorization scale. The photon participates by direct coupling to quarks or to a quark or a gluon produced through a QCD evolution starting from a bound state or to a perturbative $q \bar{q}$-pair state. There are three terms: the direct term of Figure 1c (a QPM generator was used to describe this interaction term, analogous to the QED process $e e \rightarrow e e \mu \mu$ but with quark masses), the singly-resolved photon contribution of Figure $1 \mathrm{~d}$ and the doubly-resolved photon contribution of Figure 1e (both of which were described by the QCD-RPC model). Since the hard scattering subprocesses are considered as perturbative within QCD-RPC, a cut, 
$p_{T}^{m i n}$, on the transverse momentum of the outgoing partons has to be specified in order to separate them from the non-perturbative contribution and avoid double counting (the $p_{T}^{\min }$ cut was not implemented for QPM). Unfortunately, there is still no model which completely removes this problem. As mentioned above, the quarks and gluons are emitted from a photon through QCD evolution, starting from either a point-like or a bound-state coupling. Total separation implies the use of another quark transfer cut-off $\left(p_{T}^{0}\right)$ at the first quark-pair creation level as shown in previous papers [16,17] and in a more recent global approach [18]. Since the existing quark and gluon parametrizations do not allow such a distinction, in the present study the outgoing partons are assumed to participate in high- $p_{T}$ jet production, while the spectator partons produce "beam-pipe" jets. The latter were generated along the direction of the incoming quasi-real photons.

In the present study the values of $p_{T}$ were found greater than $1.5 \mathrm{GeV} / c$, i.e. much larger than the magnitude of the mass of the virtual photons. Within the QCD-RPC model, the $p_{T}^{2}$ of the outgoing partons of the hard scattering subprocess in multi-jet production (which is always greater than a required minimum value $\left(p_{T}^{\text {min }}\right)^{2}$ ) probes the structure of one photon in singly-resolved processes, or both photons in doubly-resolved processes [19]. In this approach the two photons need to be considered as quasi-real.

Thus the main features of the two-photon mechanism used to describe the data are the following. Most secondary particles from the $\gamma \gamma$ events are produced at small polar angles, and only some of the particles of the final system are actually detected. The resolved photons produce beam-pipe or "remnant" jets, which may mix with decay products of the low- $p_{T}$ central system. The presence of jets of (moderately) high $p_{T}$ at large angles forms a signature for the isolation of the hard scattering subprocesses from the dominant diffractive dissociation contribution.

Many partonic density functions of the photon are available, but since they are extracted from deep inelastic e $\gamma$ scattering at high $Q^{2}$ they cannot always be used to describe hard scattering processes at relatively low $\left(p_{T} / c\right)^{2}$. Only leading order parametrizations have been considered here. A priori each of these parametrizations is associated with a specific value of $p_{T}^{\text {min }}$, constrained by the description of the visible total cross-section and can be tested directly against the data. This approach, as shown below, indicates the distinctive requirements for a successful parametrization. In particular, hard parton distributions will produce more high- $p_{T}$ jets in the central region while soft distributions lead to more energy deposition in the jets from the photon remnants.

These three models (VDM, QPM and QCD-RPC) were implemented in the TWOGAM generator [20] used for generating $\gamma \gamma$ events, requiring one lepton to be scattered towards the VSAT polar angle region (see Section 3). No selection was applied to the other lepton, since it mainly goes inside the beam pipe. The generated events were then fragmented using the JETSET 7.3 [21] model. In the simulation of the VDM model, the JETSET parameter $\sigma_{q}$, describing the dispersion of the transverse distribution of primary hadrons within a jet, was set to $450 \mathrm{MeV} / \mathrm{c}$ in order to take into account the bound-state origin of the quarks [22].

To take into account the influence of the magnetic field of DELPHI and superconducting quadrupoles on the scattered leptons, specially developed programs were used for fast selection of events with a lepton hitting a VSAT module, as well as for simulating and reconstructing the resulting VSAT response. These programs have been extensively used in VSAT luminosity studies. The hadronic parts of the events were simulated and reconstructed by standard DELPHI programs.

Simulations were performed separately for the VDM, QPM and QCD-RPC processes. Five parametrizations of the parton density function of the photon were chosen for sim- 
ulation [23]: the Gordon-Storrow (GS) [24] model, the Drees-Grassie (DG) [19] model, the Duke-Owens (DO) [25] model and two of the Levy-Abramowicz-Charchula models (LAC1 and LAC3) [12].

In this analysis, the $Q^{2}$ values measured by the VSAT are of the order of $0.1\left(\mathrm{GeV} / c^{2}\right)^{2}$, much lower than the $\left(p_{T} / c\right)^{2}$ of the jets (for all the QCD-RPC models, the $\left(p_{T}^{\text {min }} / c\right)^{2}$ values are greater than $2\left(\mathrm{GeV} / c^{2}\right)^{2}$, so that the simulation can be made as for a no-tag case.

This would not be true for $Q^{2}$ values around $1\left(\mathrm{GeV} / c^{2}\right)^{2}$ since there is still no theoretical description of $\gamma \gamma$ collisions in the region where $Q^{2}$ and $\left(p_{T} / c\right)^{2}$ are of the same magnitude. The total cross-section could, in principle, be unfolded from the data with the great advantage that the uncertainty in the VDM form factor extrapolation would be small.

\section{Apparatus and Event Selection}

A detailed description of the DELPHI detector can be found in ref. [26]. Only the DELPHI components relevant to the $\gamma \gamma$-event analysis (charged-particle tracking and electromagnetic calorimetry) are briefly described here. The VSAT sub-detector [27] is described here somewhat more thoroughly.

A right-handed coordinate system is used in this paper. The z-axis lies along the electron-beam direction and the $y$-axis is defined to point vertically upwards with the origin at the nominal beam-crossing point. The polar angle $\theta$ is measured with respect to the $z$-axis, and the azimuthal angle $\phi$ is measured with respect to the horizontal plane. Charged-particle tracks are measured in a $1.2 \mathrm{~T}$ magnetic field parallel to the beam axis by three cylindrical tracking chambers: the Inner Detector (ID), covering polar angle from $30^{\circ}$ to $150^{\circ}$ at radii 12 to $28 \mathrm{~cm}$, the Time Projection Chamber (TPC), the main tracking device, covering polar angles from $20^{\circ}$ to $160^{\circ}$ and radii between 35 and $111 \mathrm{~cm}$ and the Outer Detector (OD) covering polar angles from $43^{\circ}$ to $137^{\circ}$ at radii between 198 and $206 \mathrm{~cm}$. Using the ID, TPC and OD, the momentum resolution is $\sigma(p) / p=0.0015 p$ where $p$ is expressed in $\mathrm{GeV} / c$.

Tracking in the forward $\left(11^{\circ}<\theta<33^{\circ}\right)$ and backward $\left(147^{\circ}<\theta<169^{\circ}\right)$ regions is performed by two pairs of Forward drift Chambers (FCA and FCB) in the end-caps.

Electromagnetic energy is measured in the barrel region by the High density Projection Chamber (HPC) and in the forward and backward regions by a Forward Electro-Magnetic Calorimeter (FEMC), consisting of 4522 lead-glass blocks in each end-cap and covering the polar angular regions $10^{\circ}<\theta<36.5^{\circ}$ and $143.5^{\circ}<\theta<170^{\circ}$. The HPC has nine layers of lead and gas covering polar angles from $43^{\circ}$ to $137^{\circ}$ and radii between 208 and $260 \mathrm{~cm}$.

Hadron shower energies are measured by combining the measurements from the HAdron Calorimeter (HAC) covering polar angles from $10^{\circ}$ to $170^{\circ}$, and from the electromagnetic calorimeters.

The Very Small Angle Tagger (VSAT) is the fundamental tool in this analysis, as it is used to tag the scattered lepton from the $\gamma \gamma$ interactions. The VSAT consists of four rectangular electromagnetic calorimeter modules, each $5 \mathrm{~cm}$ high, $3 \mathrm{~cm}$ wide and 24 radiation lengths deep. The modules consist of 12 tungsten absorbers, each two radiation lengths thick, each followed by an energy sampling silicon plane, giving an energy resolution of $35 \% / \sqrt{E(\mathrm{GeV})}$. Three silicon-strip planes are placed around the longitudinal maximum of the electromagnetic showers (between five to nine radiation lengths inside the modules), two measuring the horizontal position and one the vertical position. Each 
strip is $1 \mathrm{~mm}$ wide, giving a reconstructed position resolution of $200 \mu \mathrm{m}$. The four modules are placed horizontally on both sides of the beam pipe at $\pm 7.7 \mathrm{~m}$ along the beam axis from the DELPHI interaction point (see Figure 2a for definitions). At this point the beam pipe changes from a cylinder of $16 \mathrm{~cm}$ diameter to an elliptical form of $16 \mathrm{~cm}$ vertically by $12 \mathrm{~cm}$ horizontally. The modules are placed after the LEP superconducting quadrupole magnets, resulting in scattered leptons being focused vertically and defocused horizontally. The magnitude of this effect is inversely proportional to the lepton energy, resulting in a VSAT polar angle coverage from 4 to $8 \mathrm{mrad}$ for the $\gamma \gamma$ case.

The quality of the event triggering system is very important in $\gamma \gamma$ data taking due to the low multiplicity of the final state, the low particle momenta and the particles mainly being boosted into low polar angles. The VSAT takes no part in the event triggering, which is done entirely on the hadronic part of the $\gamma \gamma$ events. The main component of the barrel part of the trigger for this analysis is the coincidence of ID and OD signals, while the forward trigger is based on the coincidence of signals from the FCA, FCB and TPC sub-triggers. The component from neutral particles contributes negligibly to the $\gamma \gamma$ trigger rate.

Because of the forward-boosted shape of the $\gamma \gamma$ events, the most important part of the event triggering is performed by the forward trigger. Information on all trigger components was recorded for the $\gamma \gamma$ events in order to test the forward trigger performance. The single-track efficiency was then calculated from the redundancy of the independent triggers, leading to a trigger efficiency of more than $95 \%$ for the final $\gamma \gamma$ event sample, selected as described below.

The multiplicity and energy of charged particles formed the basic criteria for selecting $\gamma \gamma$ hadronic events. Charged particles were accepted if the following criteria were met:

- momentum larger than $0.4 \mathrm{GeV} / c$;

- polar angle from $20^{\circ}$ to $160^{\circ}$;

- radial projection of the impact parameter relative to the interaction point less than $4 \mathrm{~cm}$;

- projection of the impact parameter along the beam direction less than $10 \mathrm{~cm}$;

- relative error on momentum measurement less than 1.

All calorimetric information was included in the event selection to reject the background from $\mathrm{Z}^{0}$ decays. Taking into account the sensitivity, stability and noise performance of the calorimeters, the following minimum-energy thresholds were chosen: $0.5 \mathrm{GeV}$ for the FEMC and HPC neutral clusters, and $1.5 \mathrm{GeV}$ of reconstructed energy for unlinked calorimetric showers in the HAC.

In order to select $\gamma \gamma$ events the following criteria were applied to the hadronic system in each event:

(a) at least three charged particles in the event;

(b) total energy of the charged particles less than $12 \mathrm{GeV}$;

(c) total visible energy less than $20 \mathrm{GeV}$;

(d) invariant mass in the range between 3 and $11 \mathrm{GeV}$;

(e) net charge not more than 2;

(f) thrust value less than 0.999 .

In addition the tagged lepton was required to have:

(g) VSAT measured energy larger than $20 \mathrm{GeV}$;

(h) $\operatorname{VSAT}(\theta, \phi)$-position reconstructed.

Criterion (a) selects hadronic final states, while criteria (b), (c) and the upper limit of (d) suppress $Z^{0}$ decay background. The lower limit of criterion (d) suppresses the resonance region of the $\gamma \gamma$ interaction and, together with criteria (e) and (g), the beam-gas back- 
ground. Criterion (f) rejects the bulk of $\tau$ pairs from $\gamma \gamma$ collisions leaving a negligible remaining contribution. Criteria $(\mathrm{g})$ and $(\mathrm{h})$ select well-measured leptons with very low $Q^{2}$.

\section{Background Rejection}

Two million $Z^{0}$ hadronic events were generated and the events which could give an energy deposition in the VSAT greater than $10 \mathrm{GeV}$ were fully simulated and reconstructed. Since no event was left after the selection criteria were applied, the background to the VSAT-tagged $\gamma \gamma$ events came from purely random coincidences between two independent events: an off-momentum beam electron hitting a VSAT module and some other physical event producing a hadronic system in the DELPHI detector. The probability of such coincident, but independent, events is given by the product of the individual probabilities of accepting off-momentum electrons in the VSAT and hadronic systems in DELPHI. The hadronic system mainly came from untagged $\gamma \gamma$ events, i.e. events with both the scattered electrons going undetected into the beam pipe. Also the decays of $\mathrm{Z}^{0}$, especially those involving $\tau$ particles, as well as beam-gas interactions also contributed to the background, though to a much smaller degree.

The probability of accepting off-momentum electrons in the VSAT was estimated by studying a specially selected hadronic $Z^{0}$ event sample with a strong signal in DELPHI (i.e. events having many charged particles, high invariant mass and large energy deposition). According to simulation such events should have given no signals in the VSAT, but some nonetheless were found in the data. The dominant part of this background, $86 \%$, occurred in the two VSAT modules on the outer side of the LEP ring. The results are shown in Figure 2, which shows the VSAT distributions for these off-momentum events in a) azimuthal angle $\phi, b$ ) polar angle $\Theta$ (both reconstructed in the interaction point by track extrapolation through the DELPHI and quadrupole magnets) and c) energy normalized to the beam energy. The different energy spectra for the VSAT inner and outer modules correspond to different off-momentum beam components of the LEP machine. The distributions for the outer modules are narrower than for the inner modules, especially for $\phi$ (shifted by $\pi$ for the outer modules). This fact was used to introduce cuts in $\phi$ for the outer modules 1 and $3\left(-0.30<\phi_{1}-\pi<0.14\right.$ and $\left.-0.38<\phi_{3^{-}} \pi<0.14\right)$ in order to reject the majority of the off-momentum electrons. No cut was applied for the inner modules. The $\phi$ selection rejects $66 \%$ of all the incident off-momentum electrons.

The final probability of purely random coincidence events passing all the single tagged $\gamma \gamma$ event selection criteria corresponds to a background level of $3.8 \%$ in the final $\gamma \gamma$-event sample.

\section{Results}

Using the VDM, QPM and QCD-RPC models to generate simulated events, only events which passed all the selection criteria described above were used in order to understand the kinematics of the VSAT tagged $\gamma \gamma$ events. Figure 3a shows the absolute masses of the tagged $\left(Q^{2}=-q_{1}^{2}\right)$ and the untagged $\left(P^{2}=-q_{2}^{2}\right)$ photons (see also Figure $\left.1 \mathrm{a}\right) . P^{2}$ is strongly peaked towards very low values since the untagged leptons generally do not leave the beam pipe. $Q^{2}$ for the VSAT tagged events is very small $\left(<Q^{2}>=0.06\left(\mathrm{GeV} / c^{2}\right)^{2}\right)$, but still much larger than $P^{2}$. There is no significant difference between the generated and reconstructed $Q^{2}$ distributions. 
The remaining part of this section deals with comparisons between data and full simulation. The final data sample consisted of 491 events.

The events of the QCD-RPC models were initially generated with low values of $p_{T}^{\text {min }}$ which were then increased until, finally, the total VDM+QPM+(QCD-RPC) simulation reproduced the observed number of data events. The VDM contribution was allowed to vary by $\pm 10 \%$ in comparison with the "standard" one defined by formula (1). This was done in order to estimate the variance of the $p_{T}^{\min }$ values due to the influence of the VDM cross-section uncertainty. From Table 1 it can be seen that the variation of $p_{T}^{\text {min }}$ with the VDM contribution is rather smooth for all the parametrizations used and also that the variations are not significant, being of the order of $5 \%$.

\begin{tabular}{||r|c|c|c|c|c||}
\hline QCD-RPC Model & GS & DG & DO & LAC1 & LAC3 \\
\hline$+10 \%$ VDM & 2.04 & 1.64 & 1.67 & 2.25 & 3.07 \\
\hline$+5 \%$ VDM & 1.99 & 1.60 & 1.63 & 2.21 & 3.01 \\
\hline "standard" VDM & 1.95 & 1.56 & 1.60 & 2.17 & 2.95 \\
\hline$-5 \%$ VDM & 1.92 & 1.52 & 1.56 & 2.14 & 2.90 \\
\hline$-10 \%$ VDM & 1.88 & 1.48 & 1.52 & 2.10 & 2.83 \\
\hline
\end{tabular}

Table 1. Final $p_{T}^{\text {min }}$ values in GeV/c for the QCD-RPC models studied.

In each case there were at least 6000 finally selected simulated events. To provide comparable conditions for each QCD-RPC parametrization, the differences in the final number of events were less than $\pm 1 \%$ after all the cuts applied.

Statistical comparisons between the data and simulated distributions were performed using two independent methods: the well-known $\chi^{2}$-test and the Kolmogorov test [28]. This algorithm, though not as well-known as the $\chi^{2}$-test, has some advantages. It does not require a minimum number of entries per bin, which is useful for testing the rather small statistical data sample available. It also takes into account the signs of the differences between distributions rather than just the magnitude, which makes the test sensitive to consecutive deviations of the same sign. The measure of compatibility between two distributions is given as a probability, $\mathcal{P}$. A probability close to $100 \%$ indicates very similar histograms, and a value near zero means that it is very unlikely that the two arose from the same parent distribution. The disadvantage of the Kolmogorov test is that the returned probability $\mathcal{P}$ for binned data could be overestimated.

The $\chi^{2}$ for each distribution was defined by the formula

$$
\chi^{2}=\sum_{i=1}^{N_{c h}}\left[\frac{R_{\text {data }}(i)-R_{M C}(i)}{\sqrt{\sigma_{d a t a}^{2}(i)+\sigma_{M C}^{2}(i)}}\right]^{2}
$$

where $R_{\text {data }}(i)$ and $R_{M C}(i)$ are the contents of bins $i, \sigma_{\text {data }}(i)$ and $\sigma_{M C}(i)$ are their errors, and $N_{c h}$ is the number of histogram channels with a content greater than 20 , used as a number of degrees of freedom.

The distribution of the invariant mass of the hadronic system, $W$, is shown for the data and for the VDM+QPM model and VDM+QPM+(QCD-RPC) models in Figures 3b and $3 \mathrm{c}$, respectively. It is clear that the $\mathrm{VDM}+\mathrm{QPM}$ model cannot provide a good description of the observed invariant mass distribution. Even when the VDM crosssection was allowed to vary across a wide range either to fulfill a total cross-section or to tune satisfactorily the description of the shape, the resulting probability $\mathcal{P}$ was very small indeed for both Kolmogorov and $\chi^{2}$-tests (indicated as 0 . in Figure $3 \mathrm{~b}$ ). However, adding the QCD-RPC component with any structure function parametrization other than LAC3 
gave a good description of the data, as illustrated by $\mathcal{P}$ with the best model on top and the worst one on bottom in Figures $3 c$ and $3 \mathrm{~d}$. The clear evidence for the need of the QCD-RPC contribution is also illustrated in Figure 3d, for the tagged energy fraction.

To illustrate the compatibility of different simulated models with the data, plots of standard event variables and inclusive distributions are presented in Figure 4, while some plots of typical variables from an analysis using jets are shown in Figure 5. Jet reconstruction was performed by using the Lund cluster algorithm [21] with a maximum distance $d_{\text {join }}$ of $1.4 \mathrm{GeV} / c$, below which two clusters are allowed to join into one.

From the $\mathcal{P}$ values given on plots, some parametrizations cannot provide a satisfactory description of all these distributions, as low probabilities are obtained for some of them. Very similar results with comparable probabilities were obtained using the $\chi^{2}$-method. In agreement with results from the AMY Collaboration [3], the LAC3 model can be rejected at this stage as the worst model, giving small values of $\mathcal{P}$ for several distributions. The physical reason for this is known: the LAC3 parametrization gives an extraordinarily large gluon density in comparison with other models.

Further attempts to distinguish between the remaining parametrizations were performed using an overall $\chi^{2}$-test, when several distributions were taken into account simultaneously. The invariant-mass, tagged-energy and jet transverse-momentum distributions were chosen as the most important (and not very closely correlated) variables. The tests were also performed with a $\pm 10 \%$ variation of the VDM cross-section. The results are shown in Table 2.

\begin{tabular}{||r|c|c|c|c||}
\hline QCD-RPC Model & GS & DG & DO & LAC1 \\
\hline$+10 \%$ VDM & 1.39 & 0.07 & 0.02 & 4.29 \\
\hline$+5 \%$ VDM & 3.52 & 0.31 & 0.08 & 3.82 \\
\hline "standard" VDM & 7.05 & 0.88 & 0.20 & 2.32 \\
\hline$-5 \%$ VDM & 9.53 & 2.35 & 0.31 & 1.17 \\
\hline$-10 \%$ VDM & 11.94 & 3.79 & 0.36 & 0.32 \\
\hline
\end{tabular}

Table 2. Probabilities (\%) from the overall $\chi^{2}$-test for the QCD-RPC models studied. The number of degrees of freedom is equal to 16.

The GS, DG and DO QCD-RPC models behave similarly, their agreement with data becoming better when the VDM contribution is decreased, whereas the LAC1 model follows the opposite trend. The probability of the tests for the GS parametrization, starting from the "standard" VDM cross-section, rises above the 5\% level. The LAC1 comes close to $5 \%$ when the VDM cross-section is $10 \%$ higher than the "standard", the same being true for the DG model when the VDM cross-section is lowered by $10 \%$ from the "standard". The DO model should be rejected because the overall probability is always very low. These results are in agreement with the previous no-tag analysis from DELPHI [5].

\section{Conclusions}

For the first time at LEP, very low $Q^{2}$ single tagged events have been studied and compared with VDM+QPM+(QCD-RPC) models. The data are consistent with the predictions for quark and gluon density functions provided the QCD-RPC model is used with the GS parametrization. The LAC1 and DG parametrizations are also capable of providing a satisfactory description of the data after tuning of the VDM contribution. The DO and LAC3 parametrizations do not adequately describe the data. 


\section{Acknowledgements}

We are greatly indebted to our technical collaborators and to the funding agencies for their support in building and operating the DELPHI detector, and to the members of the CERN-SL Division for the excellent performance of the LEP collider. 


\section{References}

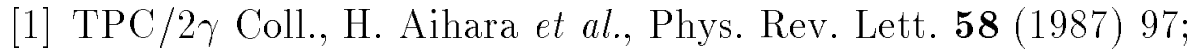

TPC/2 $\gamma$ Coll., H. Aihara et al., Phys. Rev. D41 (1990) 2667.

[2] PLUTO Coll., Ch. Berger et al., Z. Phys. C26 (1984) 353.

[3] AMY Coll., R. Tanaka et al., Phys. Lett. B277 (1992) 215.

[4] ALEPH Coll., D. Buskulic et al., Phys. Lett. B313 (1993) 509.

[5] DELPHI Coll., P. Abreu et. al., Z. Phys. C62 (1994) 357.

[6] OPAL Coll., R. Akers et. al., Z.Phys. C61 (1994) 119.

[7] L3 Coll., O. Adriani et. al., Phys. Lett. 318B (1993) 575.

[8] H.Kolanosky, Two-Photon Physics at $e^{+} e^{-}$Storage Rings, Springer Verlag, 1984.

[9] Proc. 9th Int. Workshop on Photon-Photon Collisions, San Diego 1992, eds. D.O. Caldwell and H.P. Paar (World Scientific, Singapore, 1993).

[10] R. Tanaka, in Proc. 9th Int. Workshop on Photon-Photon Collisions, San Diego 1992, eds. D.O. Caldwell and H.P. Paar (World Scientific, Singapore, 1993) p.87.

[11] M. Drees, R.M. Godbole, Nucl. Phys. B339 (1990) 355.

[12] H. Abramowicz, K. Charchula and A. Levy, Phys. Lett. B269 (1991) 458.

[13] J. Rosner, NBL report 17522 (1972) 316, unpublished.

[14] I.F. Ginzburg, V.G. Serbo, Phys. Lett. B109 (1982) 231.

[15] J.J. Sakurai, D. Schildknecht, Phys. Lett. B41 (1972) 489.

[16] C. Peterson, T.F. Walsh and P.M. Zerwas, Nucl. Phys. B174 (1980) 424.

[17] J.H. Field, F. Kapusta and L. Poggioli, Phys. Lett. 181B (1986) 362;

Z. Phys. C36 (1987) 121.

[18] G.A. Schuler and T.Sjöstrand, " $\gamma \gamma$ and $\gamma p$ Events at High Energies", CERN-TH 7193/94 (1994), to be published in Proc. Workshop "Two photon Physics from DA $\Phi$ NE to LEP200 and beyond", Paris (1994), eds. F. Kapusta and J. Parisi (World Scientific, Singapore).

[19] M. Drees, K. Grassie, Z. Phys. C28 (1985) 451.

[20] A. Nova, A. Olshevski, T. Todorov, DELPHI Note 90-35 PROG/152, unpublished.

[21] T. Sjöstrand, "PYTHIA 5.6 and JETSET 7.3", CERN-TH 6488/92 (1992).

[22] PLUTO Coll., Ch. Berger et. al., Phys. Lett. B149 (1984) 421;

TASSO Coll., M. Althoff et. al., Z. Phys. C31 (1986) 527;

CELLO Coll., H.J. Behrend et. al., Z. Phys. C51 (1991) 365.

[23] H. Plothow-Besch, "PDFLIB: A Library of all available Parton Density Functions of the Nucleon, the Pion and the corresponding $\alpha_{s}$ calculations", CERN/PPE 92-123 (1992).

[24] L.E. Gordon and J.K. Storrow, Z. Phys. C56 (1992) 307.

[25] D.W. Duke and J.F.Owens, Phys. Rev. D26 (1982) 1600.

[26] DELPHI Coll., P. Aarnio et. al., Nucl. Instr. and Meth. A303 (1991) 233.

[27] S. Almehed et al., Nucl. Instr. Meth., A305 (1991) 320.

[28] W. T. Eadie et.al, Statistical Methods in Experimental Physics, North-Holland, 1971

CERN/CN, "HBOOK Reference Manual", CERN Program Long Writeup Y250 (1992). 


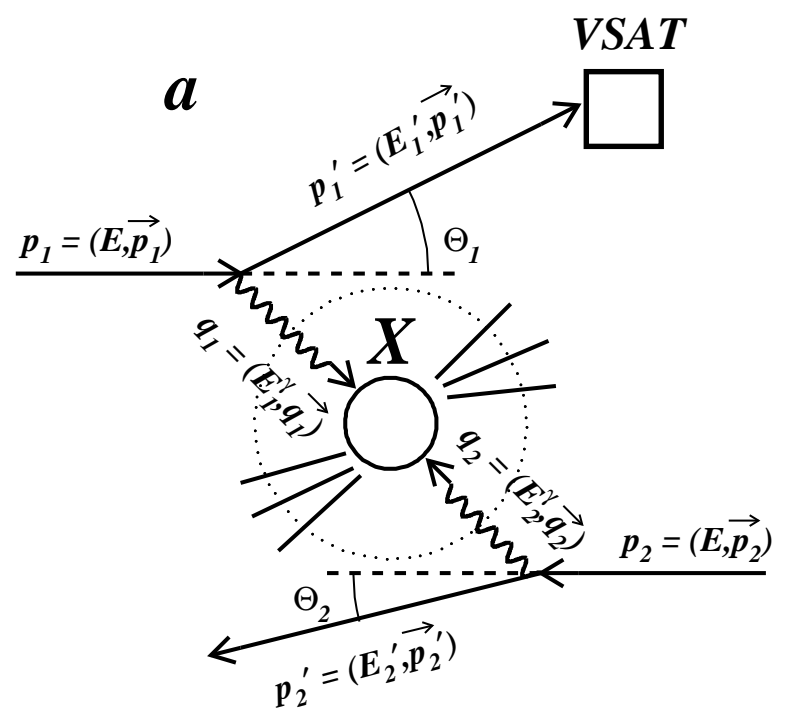

$b$
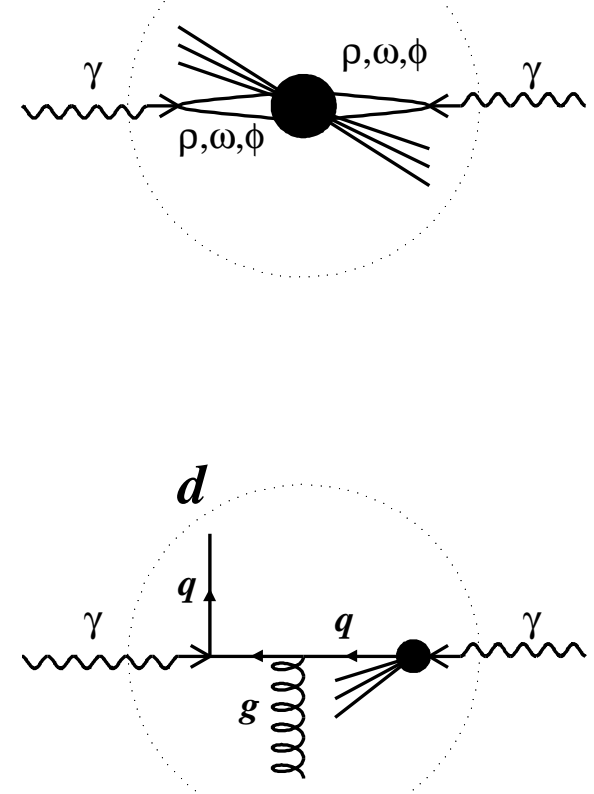

c
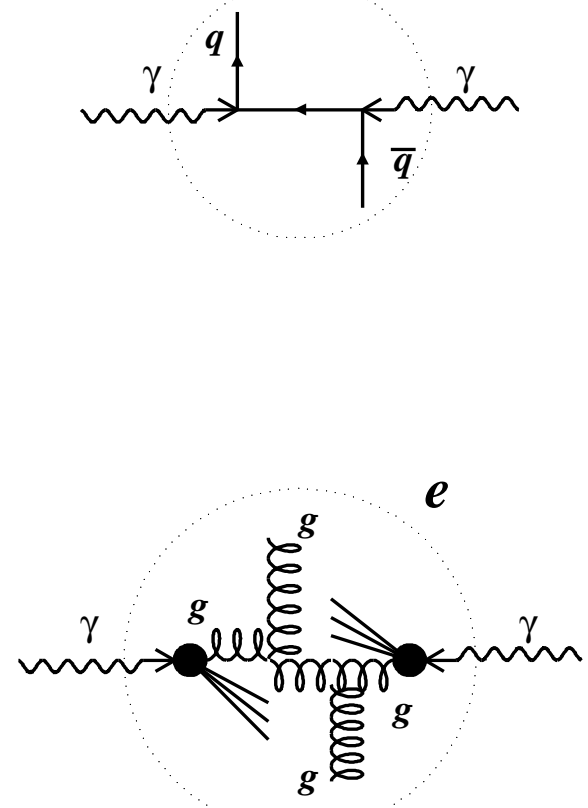

Figure 1: (a) Kinematics of two-photon reaction. One of four VSAT modules is shown as an example of a tagging detector, which measures an energy $E^{\prime}$ and an angle $\Theta_{1}$ of one of the scattered leptons. (b-e) Diagrams contributing in the lowest order to the $\gamma \gamma$ multihadronic system $X$ : (b) non-perturbative contribution (VDM); (c) direct photon contribution (QPM). Examples of QCD resolved photon contribution (QCD-RPC), (d) for singly-resolved photon and (e) for doubly-resolved photon. 

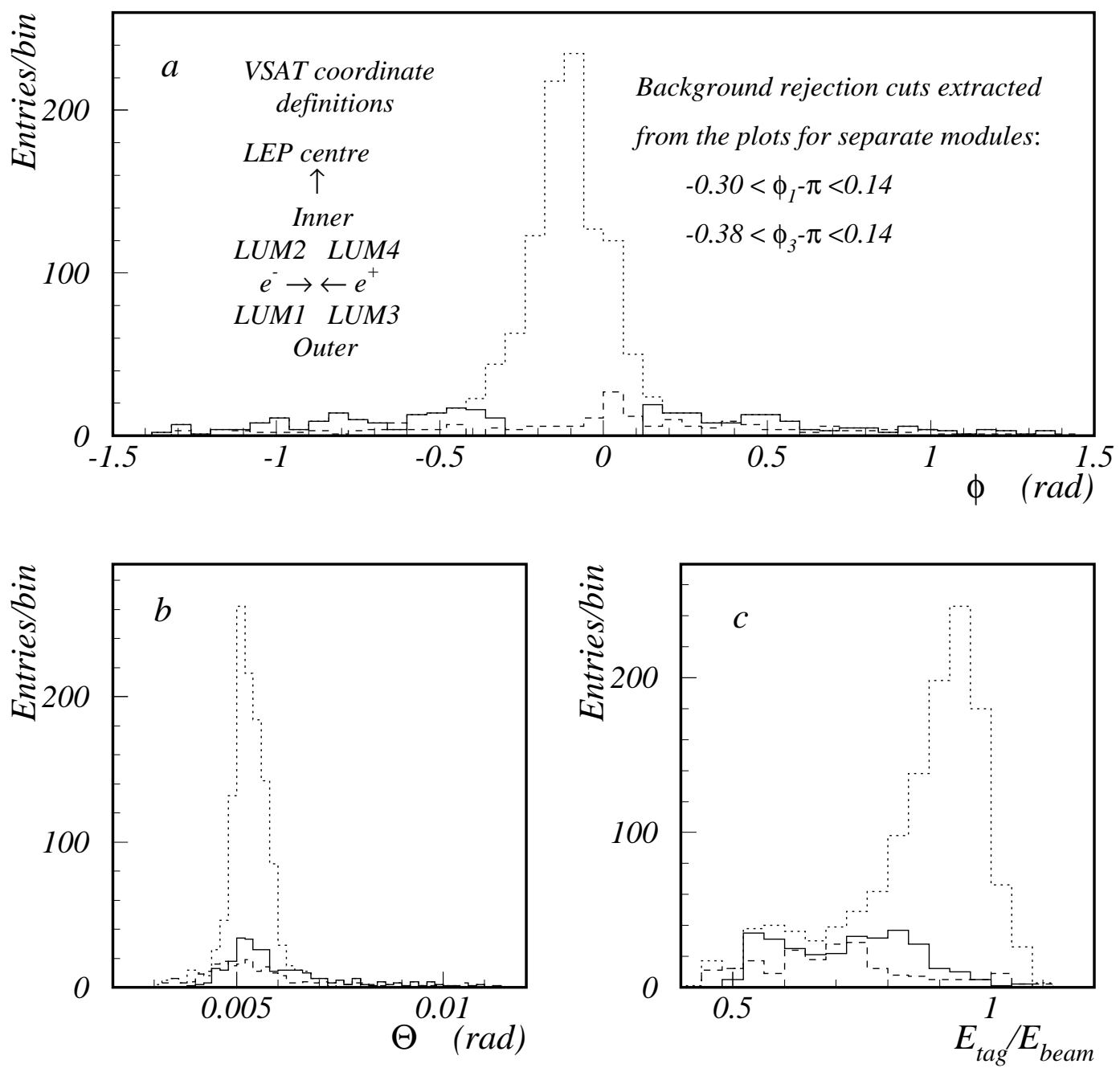

Figure 2: VSAT off-momentum electron background distributions, found from a random coincidence between well measured $Z^{0}$ events and high energy signals in one of four VSAT modules (denoted LUM1 to LUM4 in the inset) : (a) $\phi$ and (b) $\Theta$-distributions, (c) $E_{\text {tag }} / E_{\text {beam }}$. The dashed lines show the background behaviors in the inner modules 2 and 4, and the dotted lines in the modules 1 and 3 (outside of the LEP ring) before rejection. Solid lines show the remaining background after applying cuts on $\phi$ to the outer modules as indicated in (a). 

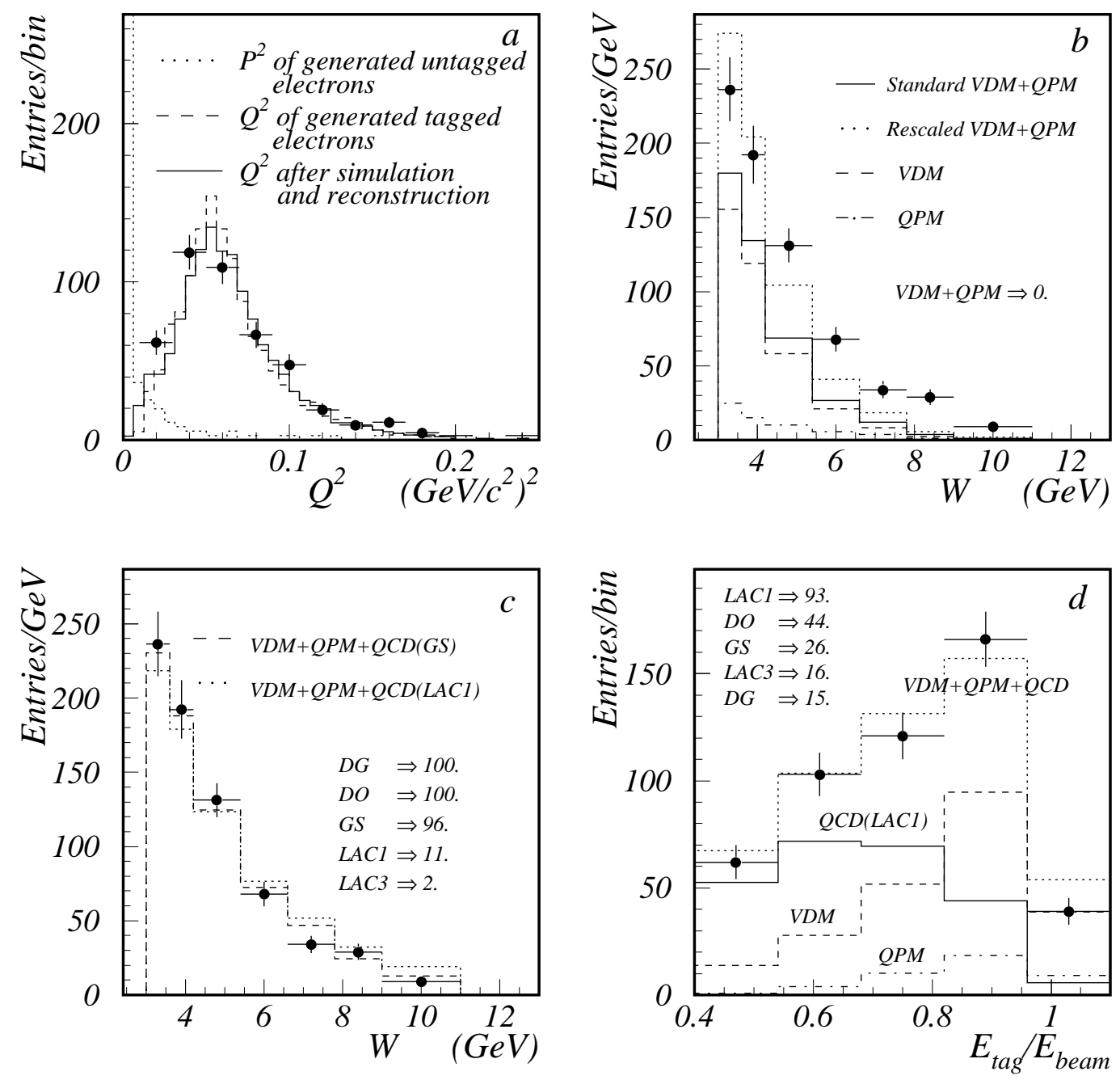

Figure 3: The black circles represent the data and the histograms the models. (a) Distribution of $Q^{2}$ for data, and histograms of generated and simulated events after final selection. (b) Distribution of the invariant mass $W$ for data and VDM+QPM models. (c) Distribution of $W$ for data and VDM+QPM+(QCD-RPC) models. (d) Distribution of tagged lepton energy, normalized to the beam energy. The inset numbers are the Kolmogorov probabilities (\%) discussed in the text. 

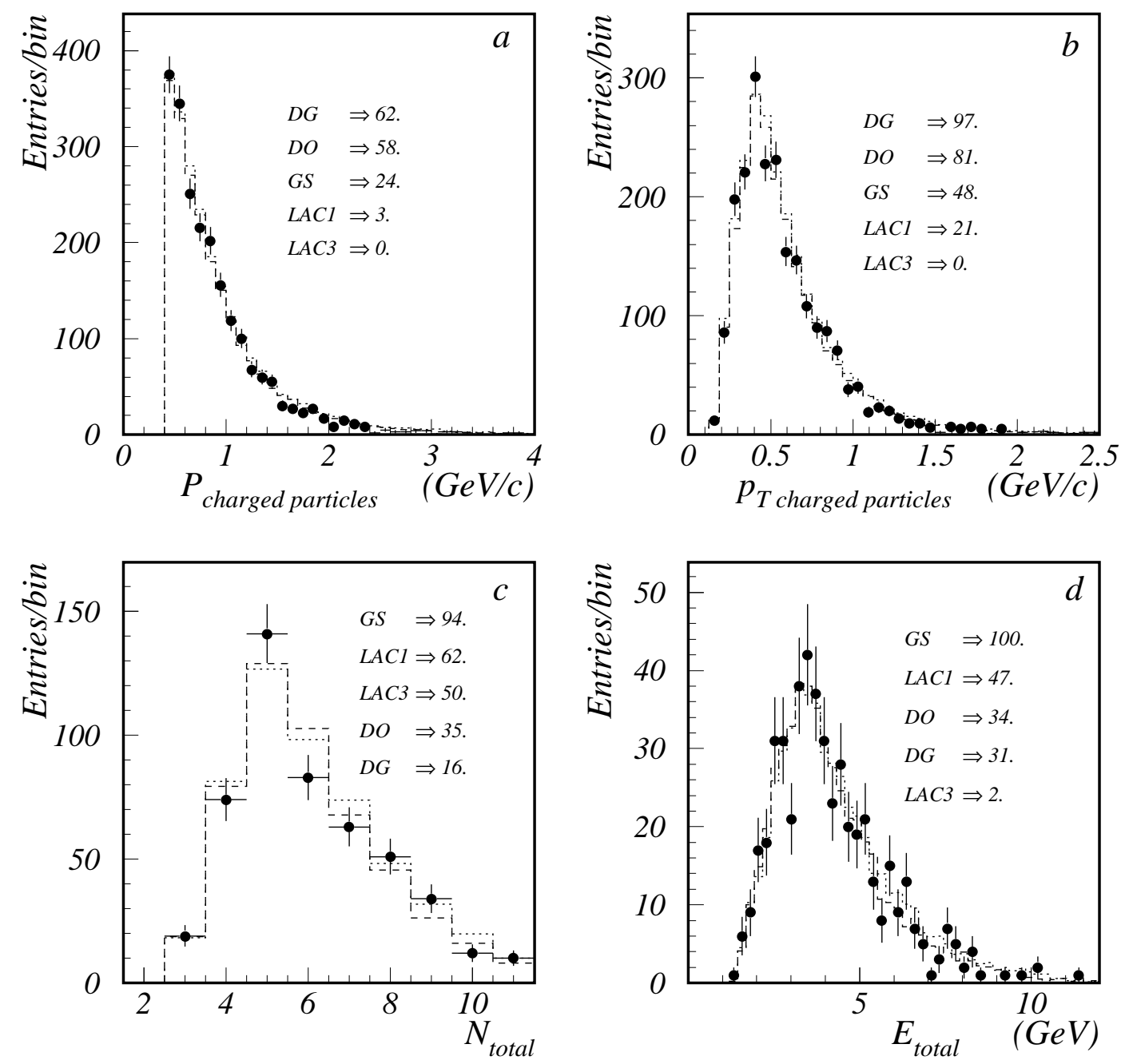

Figure 4: Comparison between data (black circles) and full VDM+QPM+(QCD-RPC) simulation. The GS parametrization used for QCD-RPC simulation is shown by dashed lines and the LAC1 parametrization is shown by dotted lines. The inset numbers are the Kolmogorov probabilities (\%) discussed in the text. (a) Charged-particle momentum. (b) Charged-particle transverse momentum. (c) Multiplicity of charged and neutral particles of hadronic system per event. (d) Total energy of charged and neutral particles of hadronic system per event. 

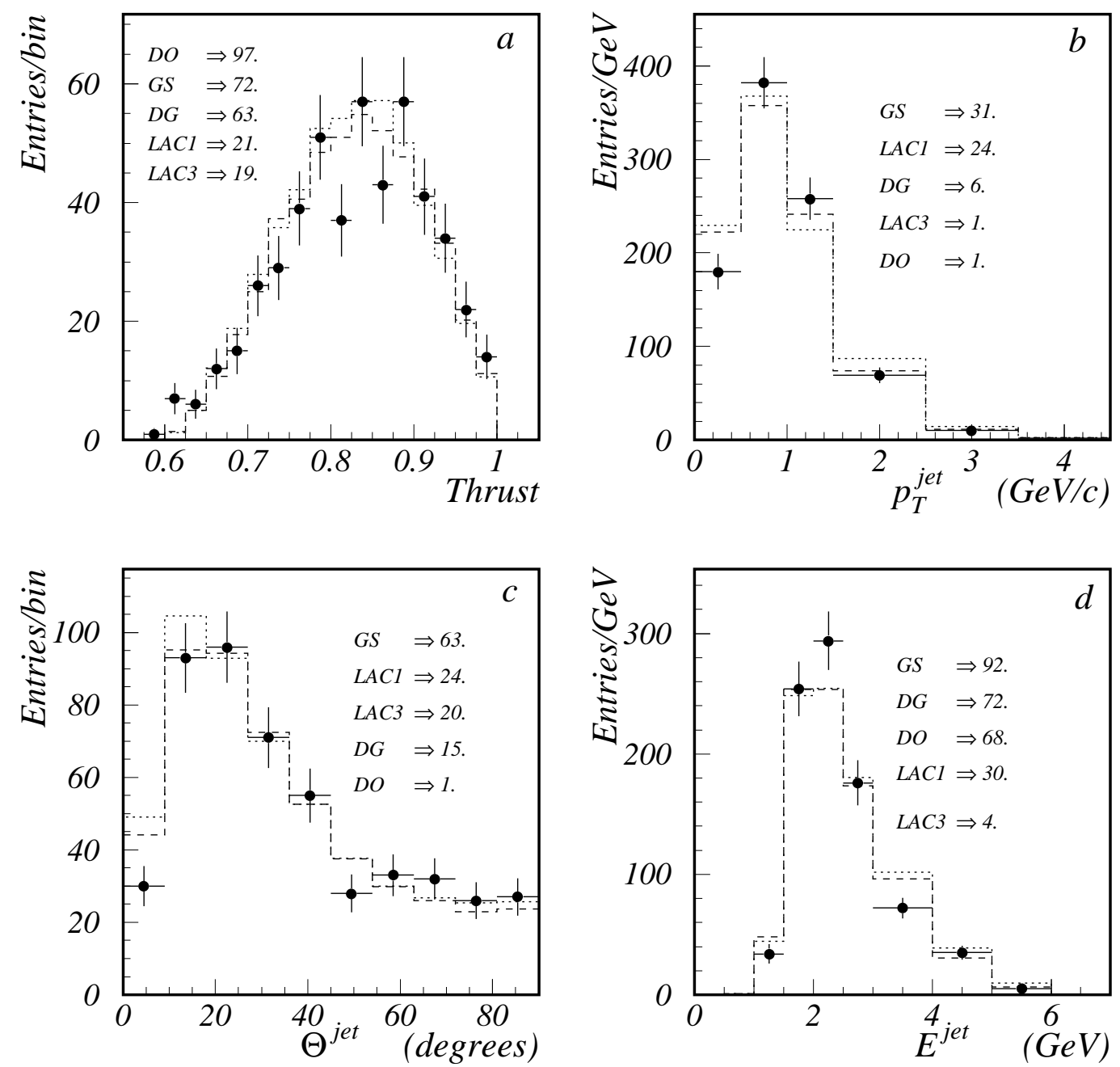

Figure 5: Comparison between data (black circles) and full VDM+QPM+(QCD-RPC) simulation. The GS parametrization used for QCD-RPC simulation is shown by dashed lines and the LAC1 parametrization is shown by dotted lines. The inset numbers are the Kolmogorov probabilities (\%) discussed in the text. (a) Thrust distribution; (b) transverse momentum, (c) angular and (d) energy distributions for the reconstructed jet with largest transverse momentum $p_{T}$. 